\title{
Efeitos da terapia manual sobre a dor em mulheres com fibromialgia: uma revisão de literatura
}

\author{
Effects of manual therapy on pain in women with fibromyalgia: a literature review
}

\author{
Marciele Monzon Ziani ${ }^{a}$, Elaine Alegre Bueno ${ }^{a}$, Lenise Romero Kippera, Fernanda Maria Vendrusculo ${ }^{\mathrm{b}}$, \\ Aline Dill Winckc, João Paulo Heinzmann-Filho ${ }^{d}$ \\ a Fisioterapeuta. Pós-graduanda em Cinesioterapia e Terapia Manual com ênfase em atendimento clínico pelo IBGEN \\ b Fisioterapeuta. Doutoranda em Pediatria e Saúde da Criança pela Pontifícia Universidade Católica do Rio Grande do Sul (PUCRS). \\ c Fisioterapeuta. Doutora em Pediatria e Saúde da Criança pela PUCRS. Professora da Universidade de Caxias do Sul (UCS). \\ d Fisioterapeuta. Doutor em Pediatria e Saúde da Criança pela PUCRS. Pesquisador do Programa de Pós Graduação em Saúde da Criança (PUCRS).
}

\begin{tabular}{l|l} 
RESUMO Objetivo: Avaliar, através de uma revisão de literatura, os efeitos da terapia manual sobre a dor em mulheres com
\end{tabular} fibromialgia (FM).

Materiais e Métodos: Trata-se de uma revisão integrativa, realizada nas bases de dados Pubmed, Lilacs, PEDro e Scielo, selecionando ensaios clínicos ou estudos quase experimental, nos idiomas português, inglês e espanhol, que objetivassem avaliar os efeitos da terapia manual sobre a dor em mulheres com FM. As palavras-chave e os operadores boleanos utilizados foram: "Musculoskeletal Manipulations OR manual therapy AND Fibromyalgia".

Resultados: De um total de 133 artigos encontrados, 9 deles foram selecionados para inclusão final no presente estudo. Desses, grande parte (55,5\%) dos estudos foram realizados no continente Europeu e apenas um foi conduzido na população brasileira. O tamanho amostral dos artigos variou de 12 a 86 indivíduos entre cada grupo estudado, sendo que $8(88,8 \%)$ deles utilizaram os critérios do Colégio Americano de Reumatologia para diagnóstico clínico da FM. As técnicas mais utilizadas foram à liberação miofascial (33,3\%), a massagem de tecido conjuntivo (22,2\%) e a drenagem linfática manual $(22,2 \%)$, sendo que a duração total do tratamento oscilou entre 3 e 20 semanas. Todos os estudos verificaram melhora da dor através das técnicas de terapia manual, com efeitos positivos também sobre os sintomas de ansiedade, sono, função física e qualidade de vida.

Conclusão: Os achados da presente revisão demonstram que as técnicas de terapia manual parecem benéficas para o tratamento da dor em mulheres portadoras de FM.

Palavras-chaves: fibromialgia; dor; terapia manual; fisioterapia.

Objective: To evaluate, through a literature review, the effects of manual therapy on pain in women with fibromyalgia (FM).

Materials and Methods: This is an integrative review study. We used the databases PubMed, Lilacs, PEDro and Scielo, selected clinical trials or quasi-experimental studies, published in Portuguese, English and Spanish, which aimed to evaluate the effects of manual therapy on pain in women with FM. The keywords and boolean operators used were: "Musculoskeletal Manipulations OR manual therapy AND Fibromyalgia". From a total of 133 articles found, 15 were selected for final inclusion in this study.

Results: Of these, most (55.5\%) of the studies were conducted on the European continent, and only one was conducted in the Brazilian population. The sample size of the studies ranged from 12 to 86 individuals for each group studied, and 8 (88.8\%) of them used the American College of Rheumatology criteria for clinical diagnosis of FM. The most widely used techniques were the myofascial release $(33.3 \%)$, connective tissue massage $(22.2 \%)$ and manual lymph drainage $(22.2 \%)$, considering that the total duration of treatment ranged between 3 and 20 weeks. All studies found pain relief through manual therapy techniques, with positive effects on the symptoms of anxiety, sleep, physical function and quality of life.

Conclusion: The findings of this review show that the manual therapy techniques seem beneficial for the treatment of pain in women with FM.

Keywords: fibromyalgia; pain; manual therapy; physical therapy.

\section{Correspondência:}

JoÃo PAUlo HeInZManN FILHO

Instituto de Pesquisas Biomédicas (IPB)

Av. Ipiranga, 6690, 20 andar

90610-000 Porto Alegre, RS, Brasil

E-mail: joaopauloheinzmann@hotmail.com 


\section{INTRODUÇÃO}

Segundo os critérios do Colégio Americano de Reumatologia, a fibromialgia (FM) é uma síndrome de etiologia desconhecida, caracterizada por dor crônica, difusa e bilateral no corpo, de característica não inflamatória e não autoimune ${ }^{1}$. Dentre as características da doença, podemse citar a sensibilidade dolorosa em sítios anatômicos preestabelecidos, a fadiga, distúrbio do sono, depressão e ansiedade ${ }^{2}$. Dados indicam que o número de pontos gatilhos relaciona-se com avaliação global da gravidade das manifestações clínicas dessa condição.

O principal sintoma e o que está presente em todos os pacientes é a dor, que pode ser difusa e crônica ${ }^{3}$. A dor geralmente começa em uma área específica, e depois se estende para todo o corpo, sendo comum a presença de ao menos 11 pontos gatilhos à palpação do total de 18 pontos conhecidos ${ }^{4}$, contudo, há pacientes que apresentam pontos dolorosos em outras partes do corpo e ainda outros que têm a doença e apresentam menos de 11 pontos dolorosos.

A prevalência da doença varia de $1 \%$ a $4 \%$ da população mundial ${ }^{4-6}(6)$. No Brasil, abrange $2,5 \%$ da população, representando a segunda doença reumatológica mais prevalente em termos de Brasil e mundo ${ }^{3}$. As mulheres são as mais afetadas pela FM e representam de 70 a $90 \%$ dos casos, sendo a maior incidência entre 35 e 55 anos $^{4,7}$.

A busca de tratamentos médicos e não farmacológicos que diminuam o impacto da FM sobre a qualidade de vida dos pacientes é fundamental para o sistema de saúde. Atualmente, o tratamento é voltado, basicamente, para a redução dos sintomas, principalmente da dor que por ser crônica torna-se um estado de saúde persistente que modifica a vida desses indivíduos ${ }^{3,8}$. De acordo com o Consenso Brasileiro de FM, os compostos tricíclicos, a amitriptilina e os relaxantes musculares, juntamente com os antidepressivos são tratamentos farmacológicos recomendados para redução dos sintomas, melhorando a dor e a funcionalidade. Já os tratamentos não farmacológicos são voltados a exercícios aeróbicos, alongamentos, fortalecimento muscular, recursos da eletroterapia, acupuntura e as técnicas de terapia manual $3,6,7,9$.

Diversos estudos abordam as técnicas manuais como importantes aliadas no tratamento da FM. Dentre as técnicas mais conhecidas estão as pompagens, a massagem terapêutica, liberação miofascial, a osteopatia e a quiropraxia ${ }^{10,11}$. Cerca de $50-75 \%$ dos pacientes com FM são tratados através dos recursos manuais, sendo a massagem frequentemente utilizada, com o objetivo de aumentar circulação sanguínea e promover o relaxamento gera ${ }^{10}$. Terapias manuais voltadas para a liberação da fáscia também têm sido investigadas para melhorar os sintomas da $\mathrm{FM}^{12}$, a combinação de tração manual e alongamentos combatem as aderências fasciais ${ }^{10}$. Recentemente, duas revisões de literatura revisaram a aplicação da neurofisiologia da dor na prática da terapia manual e as principais abordagens terapêuticas utilizadas no tratamento da $\mathrm{FM}^{13,14}$. No entanto, essas revisões não utilizaram nenhuma estratégica de busca para a seleção e inclusão dos artigos ${ }^{13,14}$. Ainda, outra revisão sistemática evidenciou efeitos positivos e moderados de diferentes técnicas de massagens sobre os sintomas e a qualidade de vida em portadores de $\mathrm{FM}^{15}$. Todavia, esse estudo não levou em conta as outras técnicas de terapia manual comumente utilizadas na prática clínica. Assim, a presente revisão adicionará informações globais, sistemáticas e abordará os efeitos de diferentes técnicas de terapia manual sobre o quadro álgico desses pacientes.

Portanto, considerando que a fibromialgia destaca-se por ser uma doença crônica de extrema relevância em termos de saúde pública e pelo fato de existirem inúmeros tratamentos não farmacológicos, torna-se importante revisar de forma crítica e sistemática os efeitos da terapia manual sobre a dor em mulheres afetadas por essa condição clínica. Esse estudo se justifica pela enorme procura fisioterapêutica para a redução da dor e pela necessidade desses profissionais conhecerem os métodos ou protocolos que apresentam maior tamanho de efeito terapêutico sobre esse desfecho estudado. Assim, o objetivo do presente estudo foi avaliar os efeitos da terapia manual sobre a dor em mulheres com fibromialgia.

\section{MATERIAIS E MÉTODOS}

O estudo consiste em uma revisão integrativa, realizada por meio de pesquisa nos bancos de dados PubMed/ Medline, Lilacs, PEDro e Scielo. Foram selecionados estudos de intervenção (ensaios clínicos) ou quasi-experimental, nos idiomas português, inglês e espanhol, sem filtro quanto ao período de publicação dos estudos. A busca foi realizada por dois pesquisadores, sendo que qualquer conflito de evidência foi definido por consenso entre ambos. O período de seleção dos estudos foi em novembro de 2015.

A busca utilizada para a seleção dos artigos foi baseada em 3 palavras-chaves, associadas com descritores booleanos. Utilizou-se a seguinte estratégia: "musculoskeletal manipulations OR manual therapy AND fibromyalgia". Esses descritores deveriam constar pelo menos no título, resumo ou nas palavras-chaves. Também, buscaram-se possíveis estudos nas referências (grey literature) dos artigos incluídos nessa revisão que pudessem preencher os critérios de elegibilidade do presente estudo. 
Foram utilizados como critérios de inclusão estudos que objetivassem avaliar os efeitos da terapia manual sobre a dor em mulheres com idade $\geq 18$ anos com FM. Em contrapartida, foram excluídos estudos de revisão, relato de caso, carta ao editor, opinião de especialista, estudos que tenham avaliado amostra composta por homens, utilização de outras técnicas de tratamento ou que não tinham relação com o tema. Além disso, os estudos que avaliaram mulheres com outras doenças associadas, também foram excluídos.

Após a identificação dos descritores no título, resumo e/ou palavras-chaves, os artigos selecionados passaram por leitura dos resumos (abstracts) para avaliar a adequação quanto aos critérios de elegibilidade. Os estudos que apresentaram os critérios de elegibilidade predeterminados obtiveram o texto completo adquirido para análise detalhada e extração dos dados.

Foram registradas as seguintes características dos estudos: nome do primeiro autor, ano de publicação do estudo, país (origem) da coleta de dados, faixa etária, tamanho amostral, formas de diagnóstico, uso de medicamentos, grupos avaliados, intervenções realizadas, quantidade de sessões, tempo da sessão, duração do tratamento e os principais resultados encontrados.

\section{RESULTADOS}

Foram encontrados um total 133 artigos, sendo 111 no Pubmed/Medline, 10 no Lilacs, 1 no Scielo e 11 no PEDro. Destes, 11 estudos eram duplicados e 117 foram excluídos por não preencherem os critérios de elegibilidade dessa revisão. Assim, foram incluídos 9 estudos, 5 estudos selecionados através da busca eletrônica e 4 adicionados através da grey literature, que avaliaram os efeitos da terapia manual sobre a dor em mulheres com fibromialgia. A Figura 1 apresenta o fluxograma do total de artigos encontrados nas bases de dados utilizadas, bem como, os motivos de exclusão dos estudos.

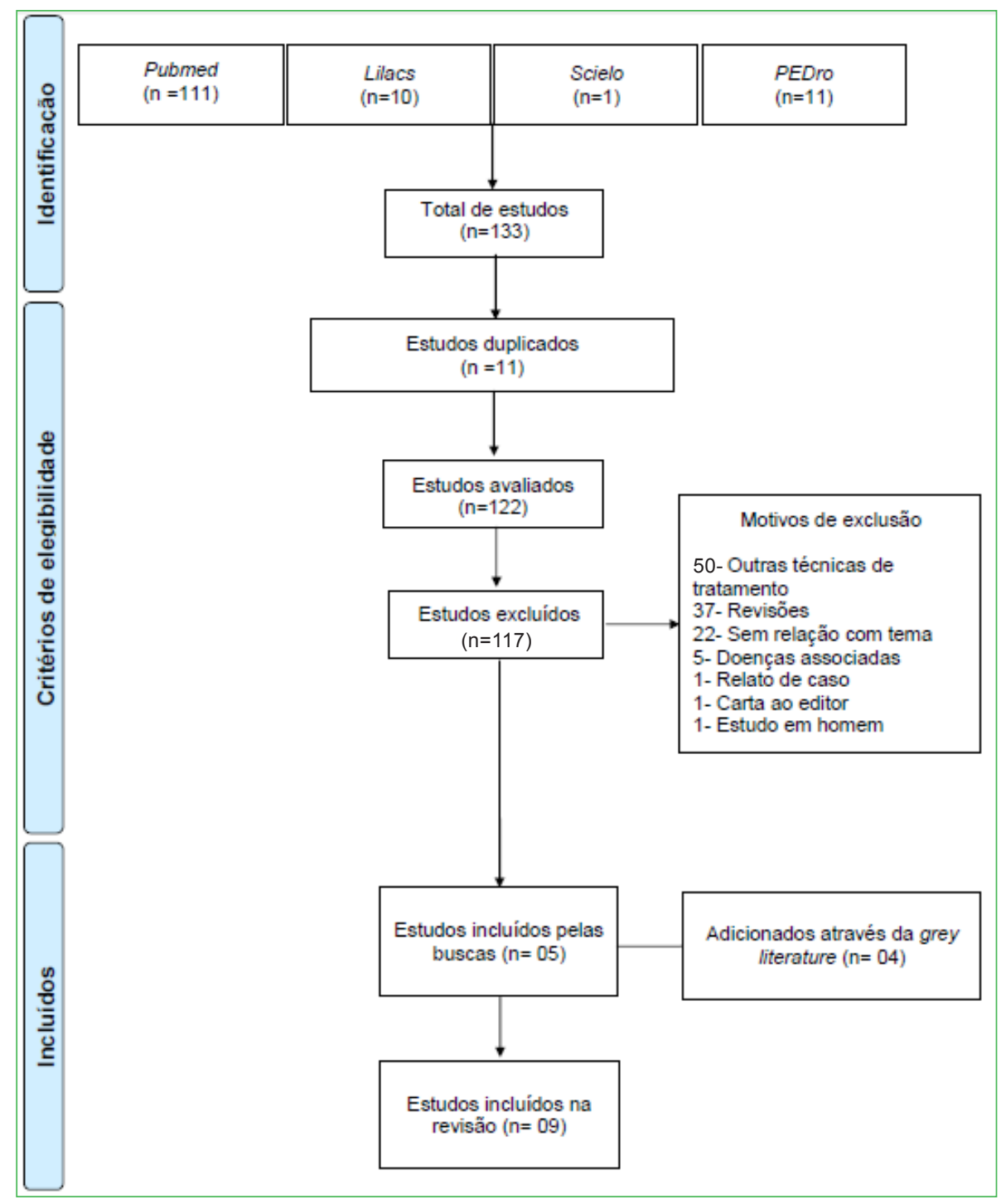

Figura 1. Sistematização da busca e seleção dos estudos. 
De um total de 9 estudos, grande parte $(55,5 \%)$ foram realizados no continente Europeu e $04(44,4 \%)$ no Americano, sendo que nesse último continente foi encontrado um estudo na população brasileira. Os artigos selecionados totalizaram uma amostra de 327 mulheres diagnosticadas com fibromialgia através dos Critérios do Colégio Americano de Reumatologia (1990), variando o tamanho amostral de 12 a 86 participantes entre os estudos. Em relação ao uso de medicação, $11,1 \%$ suspenderam os fármacos antes e durante o estudo, 22,2\% não especificaram esse item e 55,5\% não modificaram essa variável para a pesquisa. As técnicas mais utilizadas foram à liberação miofascial em 33,3\%, a massagem de tecido conjuntivo em $22,2 \%$ e a drenagem linfática manual em $22,2 \%$. Apenas $33,3 \%$ estudos utilizaram um grupo controle (placebo) para avaliar os efeitos dessas técnicas sobre a dor em mulheres com fibromialgia. Estes dados são representados na Tabela 1.

Em relação às variáveis avaliadas pelos estudos, todos avaliaram a dor de forma subjetiva por meio de escalas e questionários auto-relatados, seguidos do sono em 55,5\%, qualidade de vida em $33,3 \%$ e da ansiedade em $33,3 \%$. As intervenções quanto à liberação miofascial abordaram mais o tratamento dos pontos dolorosos com técnicas de liberação, compressão-descompressão e alongamentos. A massagem de tecido conjuntivo abordou mais algumas regiões corporais de maior tensão como área lombossacral, torácica, cervical e escapular. Já a drenagem linfática manual incluiu o uso de movimentos leves, transacionais e rítmicos em direção ao fluxo linfático abrangendo todo o corpo. Quanto a frequência das terapias, as sessões variaram entre 1 e 5 vezes na semana, sendo mais frequente 02 vezes semanais. O tempo de atendimento oscilou entre 05 e 90 minutos e a duração dos tratamentos (período do estudo) variou de 3 a 20 semanas. Todos verificaram melhora sobre a dor por meio das técnicas de terapia manual utilizadas pelos estudos. Além disso, houve melhora sobre os sintomas de ansiedade, sono, função física e qualidade de vida. A Tabela 2 demonstra as características dos estudos, protocolos utilizados e seus principais resultados.

Tabela 1. Informações gerais dos estudos incluídos na presente revisão.

\begin{tabular}{|c|c|c|c|c|c|c|}
\hline Autor \& ano & País & $\begin{array}{l}\text { Faixa etária } \\
\quad(\text { anos })\end{array}$ & $\begin{array}{l}\text { Tamanho } \\
\text { amostral }\end{array}$ & Forma de diagnóstico & Uso de medicamentos & Grupos avaliados \\
\hline $\begin{array}{l}\text { Liptan et al. } .^{10} \\
\text { (2013) }\end{array}$ & Estados Unidos & $21-50$ & 12 & $\begin{array}{l}\text { Critérios do Colégio Americano } \\
\text { de Reumatologia (1990) }\end{array}$ & $\begin{array}{l}\text { Manter inalterada a medicação no } \\
\text { período de } 3 \text { meses antes } \\
\text { do estudo }\end{array}$ & $\begin{array}{l}\text { G1: Massagem sueca } \\
\text { G2: LMF }\end{array}$ \\
\hline $\begin{array}{l}\text { Ekici et al. }{ }^{19} \\
\text { (2009) }\end{array}$ & Turquia & $\geq 25$ & 50 & $\begin{array}{l}\text { Critérios do Colégio Americano } \\
\text { de Reumatologia (1990) }\end{array}$ & $\begin{array}{l}\text { Não utilizar antidepressivos, } \\
\text { miorrelaxantes e anti-inflamatórios } \\
\text { não esteroides } 3 \text { dias do estudo }\end{array}$ & $\begin{array}{l}\text { G1: DLM } \\
\text { G2: MTC }\end{array}$ \\
\hline $\begin{array}{l}\text { Yuan et al. }{ }^{11} \\
\text { (2013) }\end{array}$ & Brasil & $33-62$ & 34 & $\begin{array}{l}\text { Critérios do Colégio Americano } \\
\text { de Reumatologia (1990) }\end{array}$ & $\begin{array}{l}\text { Manter a farmacoterapia } \\
\text { convencional durante o estudo }\end{array}$ & $\begin{array}{l}\text { G1: Shiatsu } \\
\text { G2: Controle }\end{array}$ \\
\hline $\begin{array}{l}\text { Castro-Sánchez et al. }{ }^{16} \\
\text { (2010) }\end{array}$ & Espanha & $18-65$ & 64 & $\begin{array}{l}\text { Critérios do Colégio Americano } \\
\text { de Reumatologia (1990) }\end{array}$ & $\begin{array}{l}\text { Manter a farmacoterapia } \\
\text { convencional durante o estudo }\end{array}$ & $\begin{array}{l}\text { G1: LMF } \\
\text { G2: Controle }\end{array}$ \\
\hline $\begin{array}{l}\text { Castro-Sánchez et al. }{ }^{20} \\
\text { (2011) }\end{array}$ & Espanha & $40-65$ & 86 & $\begin{array}{l}\text { Por médicos do Hospital } \\
\text { Complexo Torrecardenas }\end{array}$ & $\begin{array}{l}\text { Manter a farmacoterapia } \\
\text { convencional durante a } \\
\text { intervenção }\end{array}$ & $\begin{array}{l}\text { G1: LMF } \\
\text { G2: Controle }\end{array}$ \\
\hline $\begin{array}{l}\text { Field T et al. }{ }^{6} \\
(2002)\end{array}$ & Estados Unidos & $50,9^{*}$ & 20 & $\begin{array}{l}\text { Critérios do Colégio Americano } \\
\text { de Reumatologia (1990) }\end{array}$ & NE & $\begin{array}{l}\text { G1: Massagem terapêutica } \\
\text { G2: Terapia de relaxamento }\end{array}$ \\
\hline $\begin{array}{l}\text { Asplund et al. }{ }^{17} \\
\text { (2003) }\end{array}$ & Suécia & $18-65$ & 17 & $\begin{array}{l}\text { Critérios do Colégio Americano } \\
\text { de Reumatologia (1990) }\end{array}$ & NE & G1: DLM \\
\hline $\begin{array}{l}\text { Çıtak-Karakaya et al. }{ }^{18} \\
(2006)\end{array}$ & Turquia & $40,1 \pm 15,0$ & 20 & $\begin{array}{l}\text { Critérios do Colégio Americano } \\
\text { de Reumatologia (1990) }\end{array}$ & $\begin{array}{l}\text { Não fazer uso de medicamentos } \\
\text { durante o estudo. }\end{array}$ & $\begin{array}{l}\text { G1: MTC e ultrassom } \\
\text { combinado com estimulação } \\
\text { galvânica pulsada }\end{array}$ \\
\hline $\begin{array}{l}\text { Gamber et al. }{ }^{21} \\
(2002)\end{array}$ & Estados Unidos & $30-65$ & 24 & $\begin{array}{l}\text { Critérios do Colégio Americano } \\
\text { de Reumatologia (1990) }\end{array}$ & Manter a medicação em uso & $\begin{array}{l}\text { G1: Manipulação osteópatica } \\
\text { G2: Manipulação osteópatica } \\
\text { e orientações } \\
\text { G3: Calor úmido } \\
\text { G4: Controle }\end{array}$ \\
\hline
\end{tabular}

* Desvio-padrão não apresentado.

G1: grupo 1; G2: grupo 2; G3: grupo 3; G4: grupo 4. DLM: drenagem linfática manual; MTC: massagem do tecido conjuntivo; LMF: liberação miofascial; FM: fibromialgia; NE: não especificado. 


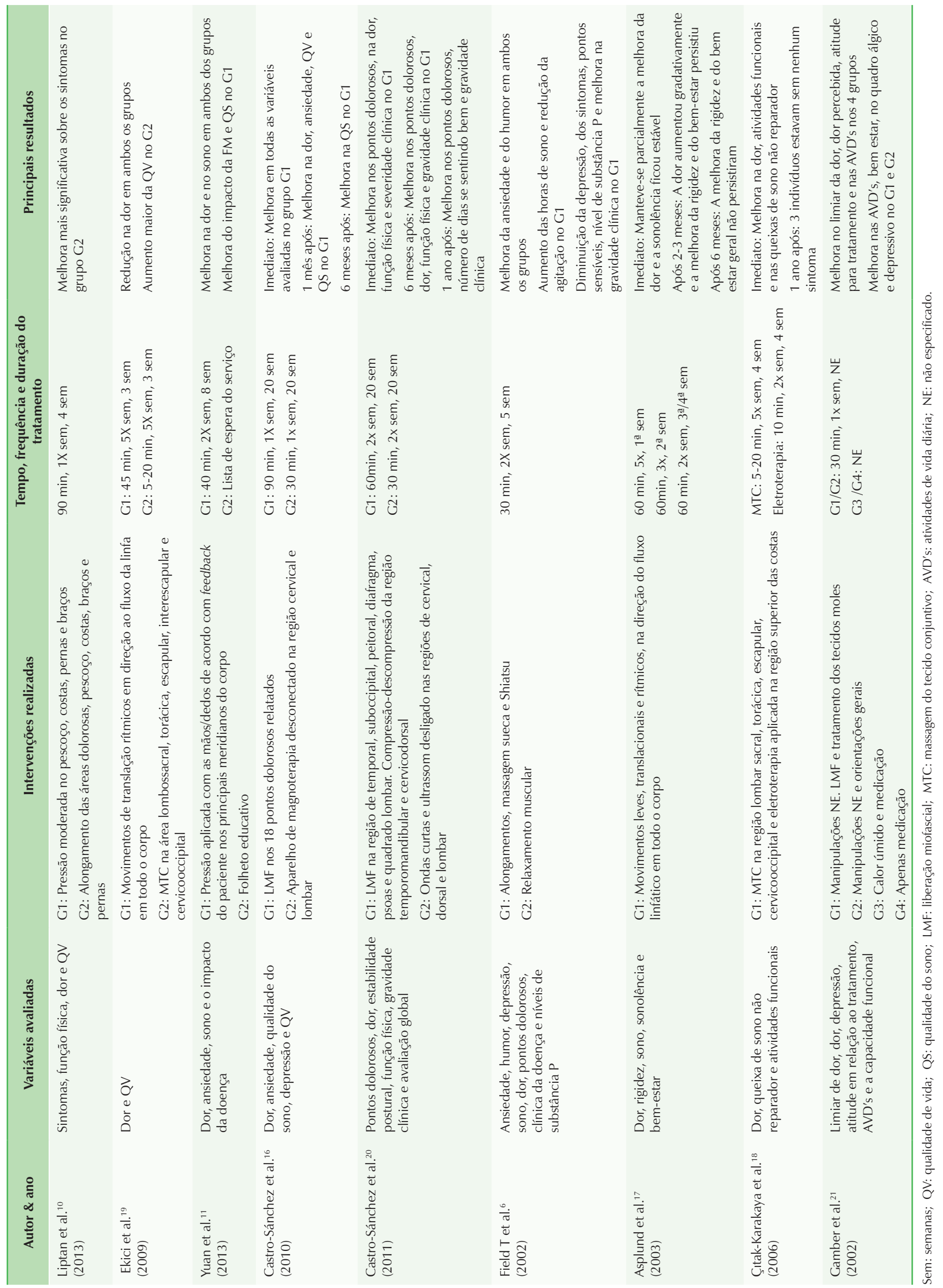




\section{DISCUSSÃO}

Na presente revisão, foram identificados 9 estudos que avaliaram a efetividade das técnicas de terapia manual sobre a dor em mulheres com FM. Desses estudos, todos obtiveram a melhora dos sintomas, principalmente a dor nas mulheres avaliadas. A terapia manual é a área dentro da fisioterapia que utiliza várias técnicas com finalidades terapêuticas, que aplicadas sobre tecidos musculares, ósseos, conjuntivos e nervosos, favorecem reações fisiológicas, promovendo a liberação de pontos gatilhos, melhora na circulação sanguínea, e relaxamento muscular ${ }^{10,11}$. As técnicas de terapia manual são compostas por vários procedimentos direcionadas às estruturas músculo esqueléticas, visando principalmente o tratamento da dor.

Os resultados dessa revisão demonstraram que a terapia manual age positivamente no quadro álgico e em outros sintomas da FM. Castro-Sánchez et al. ${ }^{16}$ em seu estudo verificou melhora da dor, qualidade do sono, ansiedade, depressão tanto imediatamente após a intervenção, como em meses seguintes utilizando a técnica de liberação miofascial. Em estudo semelhante, Asplund et al. ${ }^{17}$ utilizou a técnica de drenagem linfática manual (DLM) e obteve resultados positivos na melhora dos sintomas, sendo eles a dor, rigidez e a qualidade do sono. A maioria dos estudos incluídos nessa revisão utilizou as técnicas manuais de liberação miofascial, DLM e a massagem do tecido conjuntivo para o tratamento da FM. A terapia da liberação miofascial leva a ruptura das aderências fasciais através da combinação de tração manual e prolongadas manobras de alongamento assistidas ${ }^{10}$. Já a massagem do tecido conjuntivo produz um relaxamento geral do corpo, reduz o espasmo muscular e a sensibilidade do tecido conjuntivo $^{18}$, enquanto a DLM estimula o sistema linfático regulando o sistema imunológico, eliminando resíduos metabólicos e reduz o excesso de líquido ${ }^{19}$. Apenas quatro estudos avaliaram os efeitos da terapia manual em longo prazo sobre os sintomas da doença. Desses, todos obtiveram melhora do quadro álgico, sendo que o estudo Asplund et al. ${ }^{17}$ relataram um retorno gradativo da dor ao longo dos meses. Além disso, apenas o estudo de Çıtak-Karakaya et al. ${ }^{18}$ relataram que dos vinte indivíduos apenas três não apresentavam nenhum sintoma após 1 ano de tratamento.

De maneira geral, percebe-se que as técnicas de liberação miofascial trazem efeitos benéficos maiores a curto, médio e longo prazo sobre a dor, os sintomas da doença, a severidade clínica e a qualidade do sono desses indivíduos. Esses achados se devem ao fato de três estudos prévios verificarem efeitos superiores dessa técnica, em comparação com outros métodos manuais e/ou grupo controle ${ }^{10,16,20}$. Além disso, outras técnicas manuais, como a drenagem linfática manual e a massagem de tecido conjuntivo parecem também melhorar o quadro álgico e sintomático desses pacientes ${ }^{17,19}$. No entanto, o tamanho de efeito dessas terapias parece ser menor e não tão bem elucidado, levando em conta que os estudos que utilizaram essas técnicas não possuíam grupo controle e/ou demonstraram superioridade de uma técnica em relação à outra. Ainda, os diferentes tipos de massagem ${ }^{6,10}$ e a osteopatia ${ }^{21}$ também podem ser alternativas para o tratamento dessa doença. Porém, obteve-se uma dificuldade em investigar isoladamente os efeitos dessas terapias, devido aos grupos utilizarem um tratamento combinado com outras técnicas fisioterapêuticas. Com base nesses resultados, sugere-se que a liberação miofascial deve ser a primeira escolha para a conduta desses pacientes, visando obter um maior tamanho de efeito terapêutico. Sugere-se também, que o tratamento de portadores de FM leve em conta a frequência e duração do tratamento, considerando que a maioria dos estudos incluídos utilizou uma frequência de dois atendimentos semanais, por um período de no mínimo 4 semanas.

Dos nove estudos incluídos nessa revisão, cinco foram conduzidos no continente Europeu e quatro no Americano, sendo que nesse último continente foi localizado um estudo nacional. De acordo com esse cenário, torna-se importante a realização de mais estudos em nosso país, considerando que esta doença é a segunda condição crônica mais prevalente das doenças reumatológicas, atingindo 2,5\% das mulheres brasileiras ${ }^{3}$. Além disso, o critério de avaliação para a FM mais utilizado foi o do Colégio Americano de Reumatologia (ACR) de 1990¹. De acordo com esses critérios são necessários à presença de ao menos 11 pontos dolorosos, à palpação do total de 18 pontos descritos para o diagnóstico clínico dessa condição ${ }^{4}$. Apenas o estudo de Castro-Sánchez et al. ${ }^{20}$ diagnosticou os pacientes com FM de acordo com avaliação médica da Associação de Fibromialgia de Almeria (Espanha). No entanto, não existem detalhes quando aos critérios utilizados pelos profissionais.

Quanto ao uso de medicamentos durante o período de intervenção dos estudos, a maioria deles manteve a medicação utilizada, apenas um suspendeu o tratamento farmacológico e dois não relataram nada a respeito desse tratamento. Acredita-se que a maioria optou por não suspender o tratamento medicamentoso, pois o tratamento ideal da FM requer uma abordagem multidisciplinar com a combinação de modalidades de tratamentos não farmacológico e farmacológico ${ }^{3}$. Somando-se a isso, a 
percepção subjetiva da dor, qualidade do sono, ansiedade, depressão e qualidade de vida foram os sintomas/sinais mais avaliados nos estudos incluídos 6,10,11,16-21. No entanto, a maioria dessas variáveis foi avaliada de forma subjetiva por meio de escalas e questionários relatados ${ }^{6,10,11,16-21}$ o que pode influenciar de maneira direta nos desfechos investigados.

Embora grande parte dos estudos conduzidos utilizassem estratégias de randomização para inclusão dos pacientes, apenas dois foram controlados, um cegado, um duplo cego e três não especificaram a forma de seleção dos pacientes. Além disso, houve uma grande heterogeneidade em relação ao tamanho amostral, faixa etária da população avaliada e a duração do tratamento. Esses aspectos citados podem se constituir limitações importantes para a avaliação dos resultados encontrados, bem como dificultar o processo de comparação e interpretação dos artigos reunidos nessa revisão. Desta forma, sugere-se que novos estudos devam levar em conta esses fatores, com o intuito de melhorar o entendimento dessas intervenções sobre a dor em mulheres com FM.

Em resumo, os achados da presente revisão de literatura demonstram que as técnicas de terapia manual parecem ser benéficas para o tratamento da dor em mulheres com FM, incluindo também efeitos positivos sobre os sintomas característicos da doença. No entanto, a interpretação desses resultados e um melhor entendimento sobre o tamanho do efeito das técnicas utilizadas podem ter sido prejudicados devido à ausência de grupo controle em grande parte dos estudos incluídos.

\section{REFERÊNCIAS}

1. Wolfe F, Smythe HA, Yunus MB, Bennett RM, Bombardier C, Goldenberg DL, Tugwell P, Campbell SM, Abeles M, Clark P. The American College of Rheumatology 1990 criteria for the classification of fibromyalgia. Arthritis Rheum. 1990;33(2):160-72. https://doi.org/10.1002/art.1780330203

2. Wolfe F. The relation between tender points and fibromyalgia symptom variables: evidence that fibromyalgia is not a discrete disorder in the clinic. Ann Rheum Dis. 1997;56(4):268-71. https:// doi.org/10.1136/ard.56.4.268

3. Heymann RE, Paiva ES, Helfenstein Junior M, Pollak MF, Martinez JE, Provenza JR, Paula AP, Althoff AC, Souza EJR, Neubarth F, Lage LV, Rezende MC, Assis MR, Lopes MLL, Jennings F, Costa Araújo LRC, Cristo VV, Costa EDG, Kaziyama HHS, Yeng LT, lamamura M, Saron TRP, Nascimento OJM, Kimura LK, Leite VM, Oliveira J, Araújo GTB, Fonseca MCM. Consenso brasileiro do tratamento da fibromialgia. Rev Bras Reumatol. 2010;50(1):56-66. https://doi.org/10.1590/ S0482-50042010000100006

4. Mosmann A, Antunes C, Oliveira Dd, Neves CLM. Atuação fisioterapêutica na qualidade de vida do paciente fibromiálgico. Sci Med. 2006;16(4): 172-7.
5. Berber JDSS, Kupek E, Berber SC. Prevalência de depressão e sua relação com a qualidade de vida em pacientes com síndrome da fibromialgia. Rev Bras Reumatol. 2005;45(2):47-54. https://doi. org/10.1590/S0482-50042005000200002

6. Field T, Diego M, Cullen C, Hernandez-Reif M, Sunshine W, Douglas S. Fibromyalgia pain and substance $P$ decrease and sleep improves after massage therapy. J Clin Rheumatol. 2002;8(2):72-6. https://doi. org/10.1097/00124743-200204000-00002

7. Provenza JR, Pollak DF, Martinez JE, Paiva ES, Helfenstein M, Heymann R, Matos JMC, Souza EJR. Fibromialgia. Rev Bras Reumatol. 2004;44(6):443-9. https://doi.org/10.1590/S048250042004000600008

8. Marques AP, Matsutani LA, Ferreira EAG, Mendonça LLFd. A fisioterapia no tratamento de pacientes com fibromialgia: uma revisão da literatura. Rev Bras Reumatol. 2002;42(1):42-8.

9. Ricci NA, Dias CN, Driusso P. The use of electrothermal and phototherapeutic methods for the treatment of fibromyalgia syndrome: a systematic review. Rev Bras Fisioter. 2010;14(1):1-9. https://doi.org/10.1590/S1413-35552010000100002

10. Liptan G, Mist S, Wright C, Arzt A, Jones KD. A pilot study of myofascial release therapy compared to Swedish massage in Fibromyalgia. J Bodyw Mov Ther. 2013;17(3):365-70. https://doi. org/10.1016/j.jbmt.2012.11.010

11. Yuan SL, Berssaneti AA, Marques AP. Effects of shiatsu in the management of fibromyalgia symptoms: a controlled pilot study. J Manipulative Physiol Ther. 2013;36(7):436-43. https://doi. org/10.1016/j.jmpt.2013.05.019

12. Liptan GL. Fascia: A missing link in our understanding of the pathology of fibromyalgia. J Bodyw Mov Ther. 2010;14(1):3-12. https://doi.org/10.1016/j.jbmt.2009.08.003

13. Nijs J, Van Houdenhove B. From acute musculoskeletal pain to chronic widespread pain and fibromyalgia: application of pain neurophysiology in manual therapy practice. Man Ther. 2009;14(1):3-12. https://doi.org/10.1016/j.math.2008.03.001

14. Dommerholt J, Hooks T, Finnegan M, Grieve R. A critical overview of the current myofascial pain literature - March 2016. J Bodyw Mov Ther. 2016;20(2):397-408. https://doi.org/10.1016/j. jbmt.2016.02.015

15. Yuan SL, Matsutani LA, Marques AP. Effectiveness of different styles of massage therapy in fibromyalgia: a systematic review and metaanalysis. Man Ther. 2015;20(2):257-64. https://doi.org/10.1016/j. math.2014.09.003

16. Castro-Sánchez AM, Matarán-Pe-arrocha GA, Granero-Molina J, Aguilera-Manrique G, Quesada-Rubio JM, Moreno-Lorenzo C. Benefits of massage-myofascial release therapy on pain, anxiety, quality of sleep, depression, and quality of life in patients with fibromyalgia. Evid Based Complement Alternat Med. 2011;2011:561753. https://doi.org/10.1155/2011/561753

17. Asplund R. Manual lymph drainage therapy using light massage for fibromyalgia sufferers: a pilot study. Int J Orthop Trauma Nurs. 2003;7(4):192-6. https://doi.org/10.1016/j.joon.2003.09.001

18. Çıtak-Karakaya I, Akbayrak T, Demirtürk F, Ekici G, Bakar Y. Short and long-term results of connective tissue manipulation and combined ultrasound therapy in patients with fibromyalgia. J Manipulative Physiol Ther. 2006;29(7):524-8. https://doi.org/10.1016/j.jmpt. 2006.06.019 
19. Ekici G, Bakar Y, Akbayrak T, Yuksel I. Comparison of manual lymph drainage therapy and connective tissue massage in women with fibromyalgia: a randomized controlled trial. J Manipulative Physiol Ther. 2009;32(2):127-33. https://doi.org/10.1016/j. jmpt.2008.12.001

20. Castro-Sánchez AM, Matarán-Pe-arrocha GA, Arroyo-Morales M, Saavedra-Hernández M, Fernández-Sola C, Moreno-Lorenzo C. Effects of myofascial release techniques on pain, physical function, and postural stability in patients with fibromyalgia: a randomized controlled trial. Clin Rehabil. 2011;25(9):800-13. https://doi. org/10.1177/0269215511399476

21. Gamber RG, Shores JH, Russo DP, Jimenez C, Rubin BR. Osteopathic manipulative treatment in conjunction with medication relieves pain associated with fibromyalgia syndrome: results of a randomized clinical pilot project. J Am Osteopath Assoc. 2002;102(6):321-5. 